\title{
A Study of Cervical Smear Cytology in Large Cell Neuroendocrine Carcinoma
}

Kazuki Kanayama1, Misao Yoneda ${ }^{1 *}$, Yoshifumi S. Hirokawa', Hiroshi Imai', Masaya Fujiwara1', Hiroyasu Inada² and Taizo Shiraishi'

${ }^{1}$ Department of Pathologic Oncology, Institute of Molecular and Experimental Medicine, Faculty of Medicine, Mie University Graduate School of Medicine, Japan ${ }^{2}$ Department of Pathology, Faculty of Pharmaceutical Science, Suzuka University of Medical Science, Japan

\begin{abstract}
We examined the possibility of diagnosing Large Cell Neuroendocrine Carcinoma (LCNEC) by cervical smear cytology. The study findings suggest that if a large number of consistent cytology characteristic features specific to LCNEC are observed in the specimen, such as salt and pepper chromatin, molding arrangement of the nuclei, rosette formation and palisade-like arrangement at the periphery of cell clumps, cytological diagnosis is possible.
\end{abstract}

Keywords: LCNEC; Salt and pepper chromatin; Nuclear body; Molding arrangement; Rosette formation; Palisade like arrangement

\section{Introduction}

Cervical neuroendocrine carcinoma account for less than 5\% of all cervical tumors, and is a rare carcinoma. According to the WHO classification, it is classified into four types: typical carcinoid, atypical carcinoid, small cell carcinoma, and large cell neuroendocrine carcinoma [1]. Small cell carcinoma and large cell neuroendocrine carcinoma are associated with a poor prognosis, and it is essential to detect these tumors at an early stage. We studied the cytological morphology of a patient with cervical Large Cell Neuroendocrine Carcinoma (LCNEC), in whom we managed to establish a cytological diagnosis.

\section{Case Report}

The patient was a woman in her 60's, who presented with the chief complaint of atypical genital dysfunctional uterine bleeding. Previous medical history included histories of diagnosis of Hashimoto's disease, brain tumor and breast cancer.

History of present illness: The patient had continuous atypical genital bleeding; therefore, a pelvic ultrasound examination was performed, which revealed a tumor with a diameter of approximately $2 \mathrm{~cm}$ in the cervix. We treated the patient by surgical resection after cytological and biopsy diagnosis. The operation performed was Total Abdominal Hysterectomy with Bilateral Salpingo-oophorectomy.

\section{Result}

\section{Cytological findings}

The cervical cytology revealed a bloody background and neutrophils and lymphocytes. In addition, numerous scattered or mass of clumped middle- to large-sized atypical cells were observed. The nuclear/cytoplasmic (N/C) ratio of the cells was high; therefore, the cells looked like bare nuclei. The chromatin showed a salt-and-pepper appearance and the nucleoli were prominent (Figure 1A). Some cells with abundant cytoplasm were also observed (Figure 1B). A molding arrangement of the nuclei (Figure 1C), rosette-like arrangement (Figure 1D), palisade-like arrangement at the periphery of cell clumps (Figure 1E), nuclear lines and paired cells were also observed. A small number of carcinoma in situ (CIS) cells were also identified. Based on these findings the patient was diagnosed as having LCNEC. The ratio between the cells with LCNEC cell forms and CIS was 9 to 1 .

\section{Histological findings}

Histopathological examination of biopsy specimens revealed a dense proliferation of the tumor cells in a restiform to alveolar arrangement. The N/C ratio of the tumor cells was high, the nucleoli were prominent, and finely stippled chromatin was observed. The results of immunohistochemistry revealed positive staining for CD56 and synaptophysin (focal), and negative staining for chromogranin A.

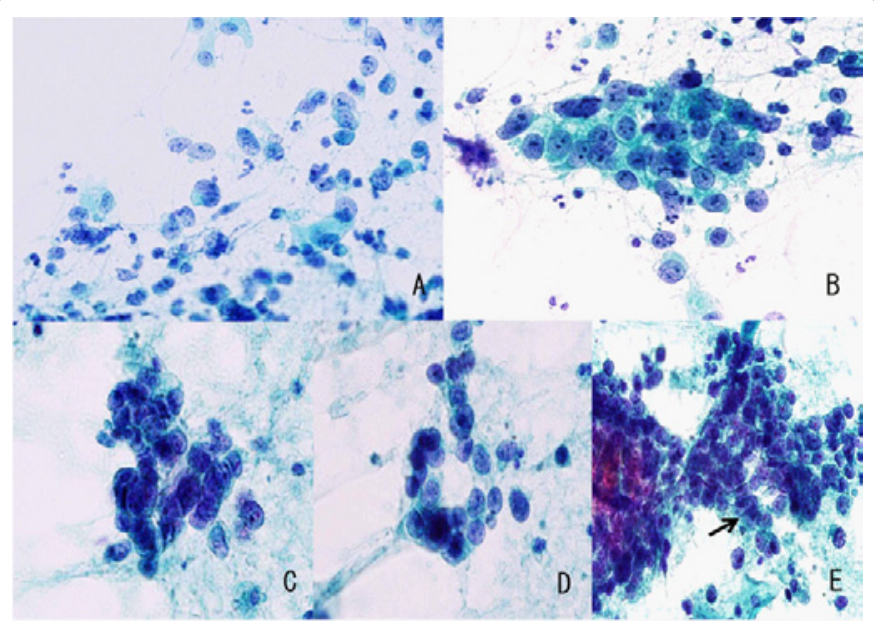

Figure 1: A: The tumor cells have scant cytoplasm with salt and pepper chromatin, and prominent nucleoli (Papanicolaou x400). B: The tumor cells have abundant cytoplasm (Papanicolaoux400). C: Molding arrangement of nuclei (Papanicolaou x400). D: Rosette like arrangement (Papanicolaou x400). E: Palisade like arrangement (Papanicolaoux400).

*Corresponding author: Misao Yoneda, Department of Pathologic Oncology, Institute of Molecular and Experimental Medicine, Faculty of Medicine, Mie University Graduate School of Medicine, 2-174 Edobashi, Tsu, Mie 514-8507, Japan, Tel: +81-59-232-2864; Fax: +81-59-231-5210; E-mail: stussykzk@clin. medic.mie-u.ac.jp

Received December 31, 2011; Accepted February 28, 2012; Published March 01, 2012

Citation: Kanayama K, Yoneda M, Hirokawa YS, Imai H, Fujiwara M, et al. (2012) A Study of Cervical Smear Cytology in Large Cell Neuroendocrine Carcinoma. Cytol Histol 3:134. doi:10.4172/2157-7099.1000134

Copyright: (c) 2012 Kanayama K, et al. This is an open-access article distributed under the terms of the Creative Commons Attribution License, which permits unrestricted use, distribution, and reproduction in any medium, provided the original author and source are credited. 
Based on these findings, a diagnosis of LCNEC was made.

Various sizes of infiltrating and proliferative lesions were observed, arranged in the shape of enhanced alveolar or trabecular structures. The tumor cells showed a salt-and-pepper appearance of chromatin and prominent nucleoli, and a number of molding and rosette arrangements were identified. Necrosis and numerous mitoses were observed. Similar to the cytology findings, the LCNEC region occupied approximately $98 \%$ of the tumor, while CIS was observed in a very limited region (Figure 2). Immunohistochemically, the tumor cells were positive for CD56 and synaptophysin (focal), and negative for chromogranin A. Metastasis lymph nodes and development of the tumor in infiltration of the tumor into the endometrium in a small area was observed. There was no evidence of tumor in the vagina. No tumor was also identified in either ovary. Based on the above, the patient was diagnosed as having LCNEC arising from the cervix.

\section{Discussion}

The diagnostic criteria of LCNEC of the cervix are the identification of, same as the case of the lung, large cells with vesicularnuclei and prominent nucleoli, a mitotic index in excess of 11/10 HPFs, and geographical necrosis $[2,3]$. Similar to the case for small cell carcinoma, LCNEC also shows a poor prognosis [4] and early diagnosis is critical. It is important to carry out imaging diagnosis using CT and MRI, as well as cytological exploration of cervical smears.

Although there have been some reports of the histopathological features of LCNEC, there have been no reports of the definitive diagnosis of LCNEC based on cytology alone. This is possibly because LCNEC is often complicated by squamous epithelial lesions and adenomatous lesions $[5,6]$, which severely reduce the appearance rate of LCNEC and also make it impossible to carry out immunostaining before the operation. In the examination of the cell appearance rate, the atypical cells of the squamous epithelial lesions and adenomatous lesions are often more prominent and the cytological diagnosis tends to be squamous carcinoma or adenocarcinoma. In our case, the LCNEC region occupied $98 \%$ of the tumor, and the CIS lesions were very limited in both the surgical specimens and cervical smears. If a large number of atypical LCNEC cells could be observed in the cervical smear, cytological diagnosis of the tumor would be possible. The definitive

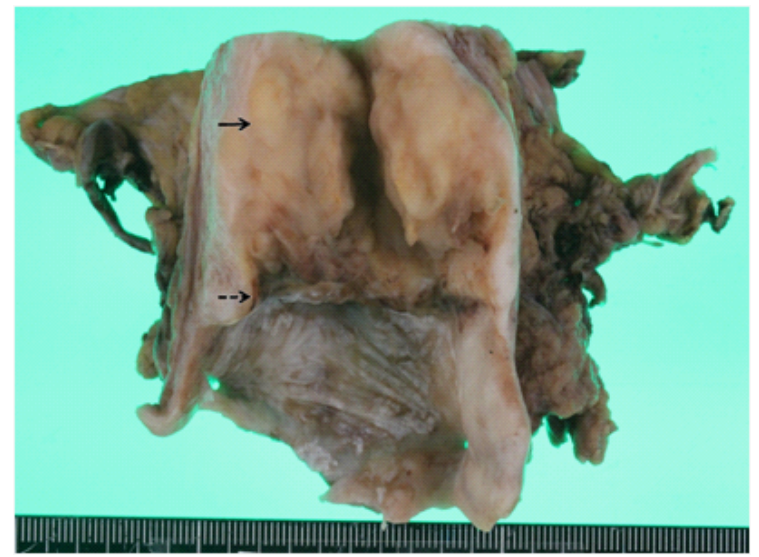

Figure 2: Cut-surface of the mass showing an yellowish-white solid tumor (solid line). CIS showed minimal range (dotted line). diagnosis of LCNEC requires confirmation of the neuroendocrine differentiation of the cells (CD56+, synaptophysin+, and chromogranin A-) by immunostaining [1]. Immunohistochemical analysis of cervical smears containing a large number of the atypical cells of LCNEC may allow definitive preoperative diagnosis of the tumor or early diagnosis. There is a need to establish an immunostaining system for cytoblock and Liquid based cytology (LBC) [7].

In regard to the cell morphology, the key is to differentiate nonkeratinizing type squamous cell carcinoma from poorly differentiated adenocarcinoma. Yamaguchi et al. listed the differential features of the former from the latter, as follows: 1) no mucus in the cytoplasm, 2) existence of nuclear lines, 3) irregular shapes of the nucleoli, and 4) smaller nucleolararea for adenocarcinoma. As another differentiating feature, they reported that the nuclei in non-keratinizing type squamous cell carcinoma show coarse granular chromatin, while the cellular nuclei in LCNEC show fine granular chromatin [8]. In our case, because of the presence of a coarse granular chromatin pattern, we suspected the patient had a non-keratinizing-type squamous cell carcinoma. However, as the features of molding arrangement, rosettelike arrangement, and palisade-like arrangement at the periphery of cell clumps were identified, we were able to make the diagnosis of LCNEC, differentiating it from other types of carcinoma. Thus, it is important to make the diagnosis by holistic examination, based not only on the observation of individual tumor cells, but also taking into consideration the tissue composition.

\section{Conclusion}

The authors encountered a patient with LCNEC, in whom the diagnosis was established preoperatively by cervical smear cytology. It can be concluded that cytological diagnosis is possible if the characteristic findings of LCNEC can be identified on cervical smear cytology.

\section{References}

1. Fattaneh AT, Peter D (2003) Pathology and genetics of tumor of the breast and female genital organs. World Health Organization Classification of Tumors.

2. Grayson W, Rhemtula HA, Taylor LF, Allard U, Tiltman AJ (2002) Detection of human papillomavirus in large cell neuroendocrine carcinoma of the uterine cervix : a study of 12 cases. J Clin Pathol 55: 108-114

3. Albores-Saavedra J, Gersell D, Gilks CB, Henson DE, Lindberg G, et al. (1997) Terminology of endocrine tumors of the uterine cervix: results of a workshop sponsored by the College of American Pathologists and the National Cancer Institute. Arch Pathol Lab Med 121: 34-39.

4. Wang KL, Wang TY, Huang YC, Lai JC, Chang TC, et al. (2009) Human papillomavirus type and clinical manifestation in seven cases of large cell neuroendocrine cervical carcinoma. J Formos Med Assoc 108: 428-432.

5. Yun K, Cho NP, Glassford GN (1999) Large cell neuroendocrine carcinoma of the uterine cervix: a report of a case with coexisting cervical intraepithelial neoplasia and human papillomavirus 16. Pathology 31: 158-161.

6. Ko ML, Jeng CJ, Huang SH, Shen J, Chen SC, et al. (2007) Large cell neuroendocrine carcinoma of the uterine cervix associated with adenocarcinoma. Taiwan J Obstet Gynecol 46: 68-70.

7. Kumagai A, Motoi T, Tsuji K, Imamura T, Fukusato T (2010) Detection of SYT and EWS gene rearrangements by dual-color break-apart $\mathrm{CISH}$ in liquidbased cytology samples of synovial sarcoma and Ewing sarcoma/primitive neuroectodermal tumor. Am J Clin Pathol 134: 323-331.

8. Tomohiko Y, Akihiko K, Hiroshi M, Shunichiro O, Keizo F, et al. (2008) Cytological study of neuroendocrine tumors of the uterine cervix -Differential diagnosis from cervical squamous cell carcinoma and adenocarcinoma. J Jpn Soc Clin Cytol 47: 86-94. 